\title{
New medical approach for rejuvenation of the periorbital area
}

\author{
Evgeniya Ranneva $^{1 *}$, Gabriel Siquier ${ }^{2}$ and Olga Liplavk ${ }^{2}$ \\ ${ }^{1}$ Dermatologist, Spain \\ ${ }^{2}$ Aesthetic Medicine Practitioner, Nederland
}

\begin{abstract}
Clinical and scientific research into new powerful products which could have potential for effective treatment in the periorbital area, and address complex problems such as lymph oedema, dark circles under the eyes and skin atrophy (wrinkles) has been ongoing for many years.

The products could be under medicine or medical device registration or license; allowing medical practitioners to perform injections in the tear trough area, palpebral malar groove and upper eyelid. The main medical proposal for this new therapeutic approach is for the final product to be safe, to deliver minimal side effects during long-term treatments, and to be clinically effective.
\end{abstract}

\section{Introduction}

Puffiness and dark circles under the eyes are very common complaints in the medical cosmetic practice. Patients, as well as their family, friends and colleagues, often see this problem as a sign of aging and tiredness. Knowledge of the anatomical structures and understanding the causes of the symptoms are prerequisites for the aesthetic medical practitioner to select optimal treatment. The purpose of this article is to provide insights into the anatomical structures and the aging process in the periorbital area; and to describe the possible treatment options using complex solutions based on non-crosslinked hyaluronic acid, exploring efficacy, possible complications and reported adverse effects.

\section{Anatomy design of the periorbital area}

The well-known anatomical classification divides the face into: upper face, mid face and lower face. At the boundary between upper and mid face is the periorbital area which also contains three zones (Figure 1).

\section{Osseous structures, innervation and vascularization}

The major osseous structure of the mid face is the cheekbones. The cheekbone is formed by the zygomatic bone laterally and the medial maxillary bone (Figure 2). An important structure in the medial part of the zygomatic bone is the infraorbital foramen that lies

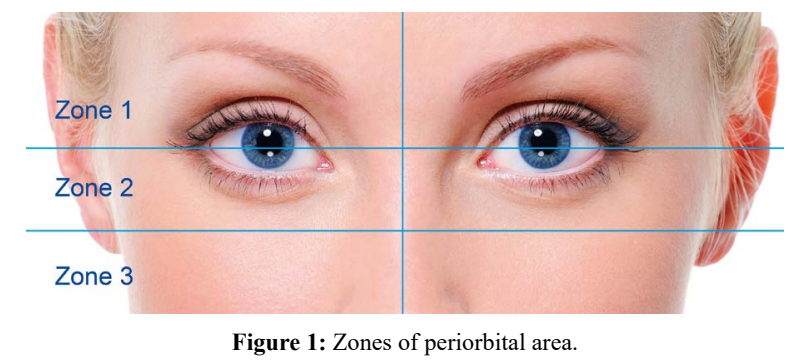

about $1 \mathrm{~cm}$ below the orbital rim mid-pupillary in the maxillary bone. Through this foramen, the infraorbital nerve and a. and v. infraorbitalis comes out of the infraorbital groove. More medially runs the a. and v. angularis, a terminal branch of the a. facial $[1,2]$. The lateral side of the cheekbone is innervated (sensorial) by the n. zygomaticus which is a branch of the $\mathrm{n}$. maxillaris (Trigeminus) and comes together with the a. and v. zigomatica from the foramen zygomaticofaciale which lies laterally in the os zygomaticum [3].

\section{Fat compartments}

The lower edge of the periorbital area forms the upper portion of

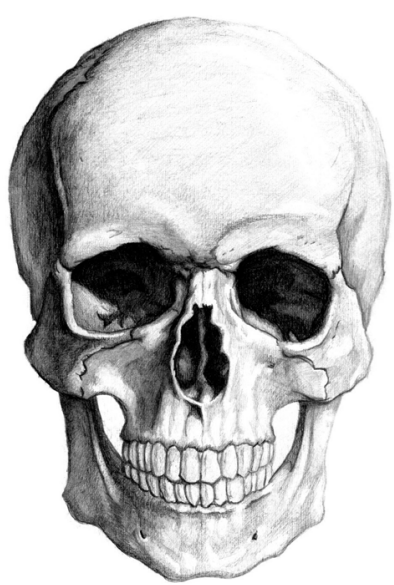

Figure 2: Osseous structure.

Correspondence to: Evgeniya Ranneva, Dermatologist, Spain, Tel: 699018934; E-mail: drranneva@clinicahera.es

Key words: eye, puffiness, eyelid

Received: June 18, 2016; Accepted: June 22, 2016; Published: June 27, 2016 
the mid face area (zone 3) and contains the deep and superficial fat pockets (Figure 3 ) which give shape and volume to the mid face area and play an important role in the formation of baggy eyes. Between those fat pockets, lies the m. Orbicularis oculi.

The deep fat compartment is fibrous, attached to os maxillaris, does not sag with aging and decrease in volume. The superficial fat tissue covers the $\mathrm{m}$. orbicularis oculi and is softer, less fibrous, stuck to the skin and moves along during expression and mimicry. The superficial fat of the cheek is made up of a plurality of compartments: medial, middle and lateral temporal $[4,5]$.

\section{Muscles}

The $\mathrm{m}$. orbicularis oculi are the most important muscle in the periorbital area and has a great relevance in the diagnosis of puffiness and circles under the eyes. This sphincter muscle is divided into three parts: pars orbital, pars lacrimal and pars palpebral is (Figure 4). The function of this muscle is closure of the eyelids, compression of the sacculus lacrimal and moving of eyebrows.

\section{Description of the aging process of periorbital area}

Chrono aging follows a predictable pattern and finds place at different anatomical levels.

The main causes of aging in the periorbital area in order of prevalence are:

- Bone reabsorption, laxity of ligaments and atrophy of the fat compartments;

- Pattern of muscles activity dramatically change from levators to depressors;

- Skin decrease the elasticity and hydration

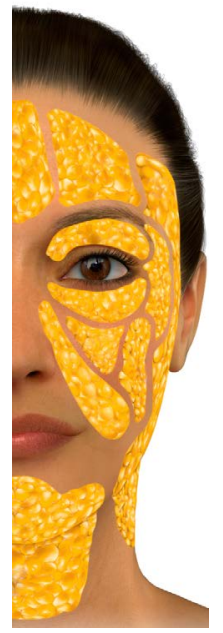

Figure 3: Fat compartments.

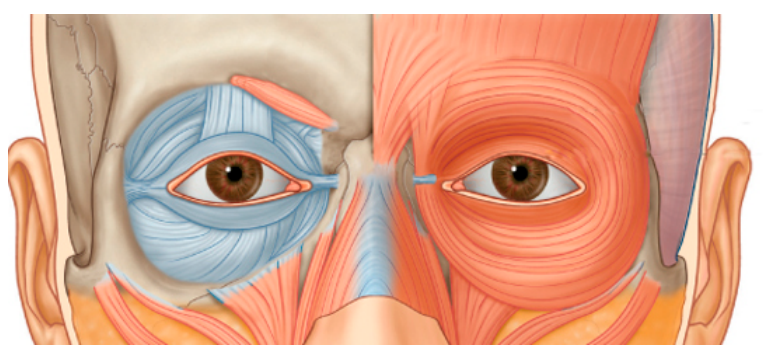

Figure 4: Muscles.
Aging process depends on or could be accelerated by several important factors such as intrinsic factors, for example genetic predisposition; and or extrinsic factors, for example UV damage. Very often Chrono aging, which is the more complex process, grows in parallel with skin photo aging (keratosis, lentigo and etc.).

The effects of the periorbital aging process manifest as loss of subcutaneous fat tissue to the forehead, brow, temple, and upper eyelid. Due to bone resorption of frontal and zygomatic bones, atrophy of the fat compartments (temporal, frontal, periorbital) and hyperactivity of $\mathrm{m}$. orbicularis, $\mathrm{m}$. procerus, $\mathrm{m}$. corrugator and $\mathrm{m}$. depressor supercilii insupercilii in zone 1, the eyebrow gets a flatter shape that creates the impression or brow ptosis, with the eyebrow seemingly descending to a position at or below the superior orbital rim. The bony outline of the skull and supraorbital rims becomes more evident, and the temporal blood vessels assume an increasingly tortuous appearance. The combination of fixed glabellar frown lines, fixed transverse forehead furrows, temporal hollowing, skeletonized supraorbital rim and a relative excess of upper eyelid skin gives the impression of upper facial aging.

In zone 3 , the os maxilla shrinks while cavitas orbitalis and apertura piriformis increase in size. The superficial and deep fat pockets of the cheek get atrophic and slides to the mid face area. The fat pockets of the middle cheek ages independently and in different speeds [6]. The increase in the glenoid orbitalis and sagging of the periorbital fat causes a palpebromalare groove (the transition of the lateral lower eyelid to the zygoma) and tear trough (transition of the medial eyelid to the maxilla) [7].

The tear trough deformity can also be seen in younger people and it is a natural phenomenon. Over time the skin in the area of the 'tear trough' gets thinner and darker than the skin of the nose and cheeks. The result is a flabby skin, visible facial expression wrinkles, discoloration and puffiness under the eyes [8].

\section{Eyes puffiness}

Baggy eyelids as well as puffiness in the eye area could have a different pathways of development:

A baggy eyelid as a result of fluid retention is very common and frequently reported. Fluid retention under the eyes occurs as a result of underlying causes such as stress, fluid retention, regular daily alcohol excess, chronic lack of sleep, insufficient moisture and impaired lymphatic drainage. In addition, treatment with Botulinum Toxin A (which has become very popular in aesthetic medicine) in the caudal part of the m. orbicularis oculi lead can reduce the pump function of the orbicularis oculi together with underlying lymphatic network, resulting in edema (eyes puffiness). Very rarely eyes puffiness can be caused by compression of the lymph path with excessive crosslinked hyaluronic acid injections in the malar area. Baggy eyelids due to sagging of periorbital fat pockets is the most well-known symptom of moderate aging in patients of both genders with positive genetic anamnesis. Baggy eyelids due to sagging of the periorbital fat pockets are caused by a weaker $\mathrm{m}$. orbicularis oculus, causing a herniation of the retro-ocular fat that can be increased by the growth of cavities orbitalis. Baggy eyelids by volume loss could affect patients of any age. The appearance of 'Tear trough' and palpebral grooves give a tired look and makes the face seem much older. The 'tear trough' is caused by loss of volume under the maxilla and it runs out to the lateral side of the face [8]. This volume loss is caused by decreasing of the bone structure support, skin aging and weakening of the orbital septum. Eventually the 
superficial fat tissue drops caudal resulting in characteristic grooves.

\section{Panda symptom}

Infraorbital dark circles (panda symptom) refers to a condition that is characterized by darkness of the infraorbital eyelids thus it can be a significant aesthetic problem. This condition affects individuals of all ages, sexes and is more frequent in the middle eastern morph type.

Excessive pigmentation under the eyes can be a frequent cause of dark circles. It can be caused by dermal monogenesis or post inflammatory hyperpigmentation as result of chronic hypoxia. Also the thinning skin folds more easily due to mimics and loss of elastin and collagen, making the pigment coloration intensely visible. The reasons for that are the anatomical characteristics of the periorbital area: thin skin, loss of firmness and elasticity, repeated facial expressions, and sensitivity to sun.

Another common cause of infraorbital dark circles can be thin, translucent lower eyelid skin overlying orbicularis oculi which is a muscle that lies just beneath the skin, with little or no subcutaneous fat over it. Therefore, a bluish discoloration may be generated by the prominence of venous capillary network [9].

Skin laxity or dermatochalasis of the lower eyelid is one of the causes of dark circles and puffiness. This is caused by a loss of elasticity in the connective tissue supporting the structure of the front portion of the eyelid. Dermatochalasis and periocular rhytides are the most common signs of area aging. Over time, collagen and elastin in the thin tissue of the eyelids and periorbital skin undergo ultraviolet-induced and age-related degeneration. In addition, the damaged epidermis releases collagenases, which contribute to collagen degradation. Skin laxity due to photo aging presents as a shadowing appearance of the lower eyelids that results in infraorbital dark circles [10].

\section{History, examination and diagnosis}

The correct diagnosis is essential for the correct treatment. These four questions should be answered: what is the cause of the complaint, is there a skin problem, is there a loss of volume or is there a surgical problem. A focused anamnesis, examination and diagnostics will answer these questions. A focused anamnesis is necessary to exclude the pre-existing health conditions: allergy, skin diseases, pregnancy, systemic disorders or anticoagulant use should be queried. As a rule, only ASA I patients should be treated. When examining the three zones of the periorbital area necessary to observe and study the skin condition (relief of the wrinkles and folds, structure of the skin; hyper/ hypopigmentation; mimic muscle activity and tonus (hypotonia, hypertonia, muscle weakness: Bell's palsy, signs of stroke), individual anatomy of the bone structure, fat pockets to diagnose volume loss or replacement [11].

\section{Actual treatments and new medical approach}

Depending on the cause of the complaint treatment can include one of these therapeutics: chemical peeling; lasers, including Q-switched ruby laser, alexandrite or Nd: YAG [8]; botulin toxin, cross-linked or non-crossed linked hyaluronic acid injections, to Figureal pharmaceutical or cosmetic products $[12,8,13]$.

From 2013 we studied a new medical device class III injectable, based on non-cross link hyaluronic acid: RRS ${ }^{\oplus}$ HA Eyes. The RRS ${ }^{\circledast}$ HA Eyes formula was designed for superficial and deep dermal injections. It is based on the purest and most effective ingredients formulated with synergistic actions. Non cross-linked HA from a biotechnological non-animal origin provides: antioxidant effect, turnover stimulation \& matrix reorganization. Every ampoule of $1,5 \mathrm{~mL} \mathrm{RRS}^{\oplus} \mathrm{HA}$ Eyes contains HA, non-cross-linked $5,0 \mathrm{mg} / \mathrm{mL}$ associated with active biorevitalization solution (BS $27,3 \mathrm{mg} / \mathrm{mL}$ ) and helps improving the transport function of the actives from BS. The complex actives of RRS HA Eyes are:

- Antioxidants: Vit C, Naringin, Resveratrol, R-2 Viniferin, $\varepsilon$-Viniferin, R-Viniferin, Iso- $\varepsilon$-Viniferin, etc.

- Flavonoids: Troxerutin, Rutin, Naringin, Hesperidin Methyl Chalcone, Hesperidin.

- Saponins: Ruscogenin, Neoruscogenin, Escin.

- Polyphenols: Resveratrol, R-2 Viniferin, $\varepsilon$-Viniferin, R-Viniferin, Iso- $\varepsilon$-Viniferin, Coumarin.

- Peptides: DipeptideVW, Peptide Pal-GQPR.

- Trace element: Organic Silicium.

These groups of nutritional supplementation that include vitamins, minerals, and/or antioxidants may help in various different ways. Vitamins are necessary components and play important roles in cellular metabolism. They are considered "micronutrients" and occur in only very small amounts within cells, but are critically important as coenzymes. Peptides have several functions: the energy storage function (proteins can be degraded into acetyl-CoA and "cycle" the Krebs cycle), the endocrine integration function (hormones), and the informative function (membrane receptors, intracellular signals). However, the biggest group of actives present in the biorevitalization solution of RRS ${ }^{\circ} \mathrm{HA}$ Eyes is antioxidants. The mechanisms by which these antioxidants act at the molecular and cellular level include roles in gene expression and regulation, apoptosis, and signal transduction. Antioxidants are involved in fundamental metabolic and homeostatic processes and help repairing damaged biomolecules and defense antioxidant enzymes, which are mostly intracellular.

RRS ${ }^{\circledR}$ HA Eyes: treatment protocol

All the patients ( 12 females and 5 males from 27 to 62 years old), were photographed with the same settings and adequate lighting. Written informed consent was taken. The authors preferred to inject with the patient reclined at $45^{\circ}$ as the tear trough deformity is better visible in this position than when the patient is lying down. Good lighting is extremely important for better visibility of anatomical landmarks. The injections should not be given in a hurry as chances of hitting blood vessels or injecting superficially are higher when in haste.

Any cosmetic makeup in the area to be treated is completely removed with a cleansing lotion. Further disinfection is done with chlorhexidine in $70 \%$ alcohol. The orbital rim is palpated. Due to the rich subdermal vascular plexus, the tear trough area is prone to significant bruising. Hence, care is taken to inject away from any visible blood vessels in this area. RRS $^{\oplus}$ HA Eyes is injected with a 32-gauge needle. RRS ${ }^{\oplus}$ HA Eyes is deposited with subdermal papula technique. Usually about $0.05-0.1 \mathrm{~mL}$ is adequate per depot. The area is gently massaged for an even distribution of the product. However, vigorous massage should be avoided in this area. The patients are asked to avoid any massage or facial treatments for $48 \mathrm{~h}$.

Some patients presented untoward effects like swelling and ecchymosis that resolved within 24 and 48 hours. Patients should avoid strong or extended pressure within the treated area. Patients should be informed about after-care goals: avoiding massage, strenuous physical 
activity and exposure to extreme cold or heat for up to six hours posttreatment.

The patients were asked to attend for follow up in 15 days and one month to see if a touch-up is required. (Figures 5-9). Observation showed that RRS ${ }^{\oplus}$ HA Eyes injections in the tear trough area usually lasts for up to 2-4 months.

\section{Conclusion}

The result of the study indicates that because of the various factors causing aesthetic problems in periorbital area, it is essential first to identify the underlying cause to provide an appropriate treatment.

The loss of the subcutaneous tissue and bone reabsorption in the subtalar area can be restored with crosslinked and non-crosslinked hyaluronic acid. In this challenging area frequently observed complications and side effects of treatment with cross-linked HA are Tyndall effect, edema and eventually overcorrection that can have a negative aesthetic effect for a long period of time. Another treatment option of the aesthetic problems at the periorbital area such as lymphedema, dark circles and volume loss around orbital rim is RRS ${ }^{\oplus}$ HA Eyes.

The results of the study indicate that injections of this product applied within the tear trough, the palpebral malar groove and also the upper eyelid area is a safe and effective treatment with minimal
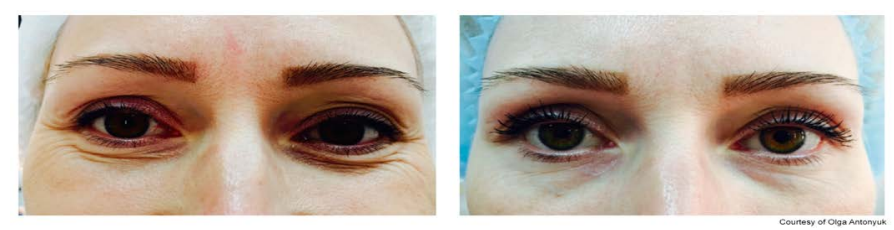

Figure 5: Results.
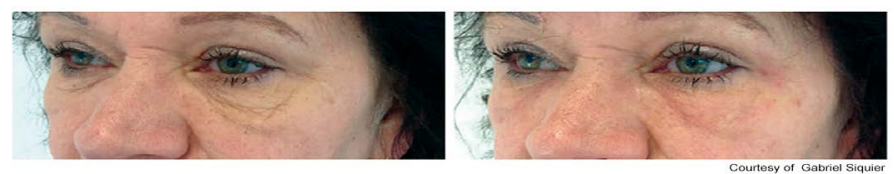

Figure 6: Results.
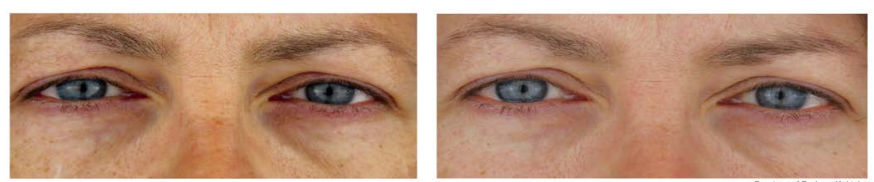

Figure 7: Results.
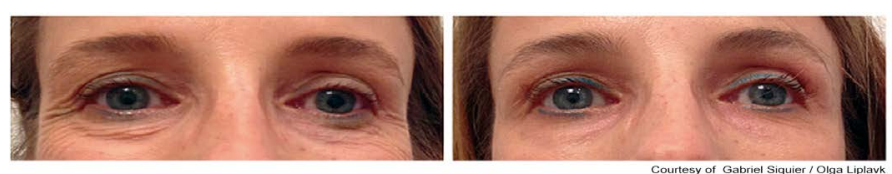

Figure 8: Results.

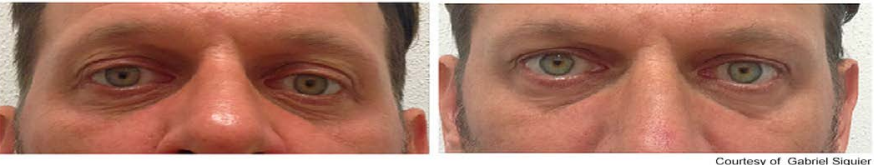

Figure 9: Results.

side effects. RRS ${ }^{\circledast}$ HA Eyes did not cause edema, no overcorrection was reported and no Tyndall effect occurred if correctly injected. The greatest efficacy was observed in case of tear trough and palpebral groove volume loss combined with lymphedema.

The biological and pharmacological functions of RRS ${ }^{\circledast}$ HA Eyes has not yet been fully investigated, but during 2015 an in vitro study to evaluate the inhibitory activity of a compound on the angiotensin enzyme was done at the University of Barcelona, this study demonstrated the positive influence on lymphangion contraction. This in vitro study could explain the visible clinical improvement of the area due to lymphatic drainage activation. Although these results with RRS ${ }^{\circledR}$ HA Eyes treatment are very satisfactory, they require further investigation with a larger patient cohort.

\section{References}

1. Tan SR, Glogau RG (2008) Fillers esthetics. In: Carruthers J, Carruthers A. Procedures in Cosmetic Dermatology: Soft Tissue Augmentation. New York: Elsevier-Saunders pp: 11-18.

2. Gosling JA, Harris PF (1958) Atlas of Human Anatomy with integrated text; Manchester University Department of Anatomy; Grower Medical Publishing Ltd.

3. Putz R, Pabst R (2016) Sobotta Atlas of Human Anatomy; Head, neck, upper limb

4. Rohrich RJ, Pessa JE (2007) The fat compartments of the face: anatomy and clinical implications for cosmetic surgery. Plast Reconstr Surg 119: 2219-2227. [Crossref]

5. Small R, Hoang D (2012) A practical guide to dermal filler procedures; Wolters Kluwer, Lippincott Williams \& Wilkins 48.

6. Belhaoiari L, Teisseire P (2012) Vectors for volumisation enhancing the mid-face with hyaluronic acid; Primp 28-40.

7. Lambros VS (2007) Hyaluronic acid injections for correction of the tear trough deformity. Plast Reconstr Surg 120: 74S-80S. [Crossref]

8. Vedamurthy M; IADVL Dematosurgery Task Force (2008) Standard guidelines for the use of dermal fillers. Indian J Dermatol Venereol Leprol 74 Suppl: S23-27. [Crossref]

9. Lafaille P, Benedetto A (2010) Fillers: contraindications, side effects and precautions. $J$ Cutan Aesthet Surg 3: 16-19. [Crossref]

10. Sadick NS, Bosniak SL, Cantisano-Zilkha M, Glavas IP, Roy D (2007) Definition of the tear trough and the tear trough rating scale. J Cosmet Dermatol 6: 218-222. [Crossref]

11. Hirsch RJ, Narurkar V, Carruthers J (2006) Management of injected hyaluronic acid induced Tyndall effects. Lasers Surg Med 38: 202-204. [Crossref]

12. Roh MR, Chung KY (2009) Infraorbital dark circles: definition, causes, and treatment options. Dermatol Surg 35: 1163-1171. [Crossref]

13. Deprez P (2016) Text book of chemical peels. Informa healthcare: 392.

Copyright: (2016 Ranneva E. This is an open-access article distributed under the terms of the Creative Commons Attribution License, which permits unrestricted use, distribution, and reproduction in any medium, provided the original author and source are credited. 